\title{
PAPR reduction of OFDM using invasive weed optimization-based optimal peak reduction tone set selection
}

\author{
Ho-Lung Hung ${ }^{1}$, Chung-Hsen Cheng $^{2}$ and Yung-Fa Huang ${ }^{3^{*}}$
}

\begin{abstract}
Tone reservation (TR) is one of the attractive techniques to reduce peak-to-average power ratio (PAPR) in orthogonal frequency division multiplexing system. As conventional TR technique requires exhaustive searching over all the combinations of the given peak reduction tone (PRT) sets, it results in computational complexity that increases exponentially with the number of the subcarriers. In this paper, we aim to obtain a desirable PAPR reduction with low computational complexity. Since the process of searching the optimal PRT set can be categorized as combinatorial optimization with some variables and constraints, we propose a novel scheme, which is based on a nonlinear optimization approach named as invasive weed optimization method, to search the optimal combination of PRT set with low complexity. To validate the analytical results, extensive simulations have been conducted, showing that the proposed schemes can achieve significant reduction in computational complexity while keeping good PAPR reduction. As results of simulations, the proposed scheme shows almost the same PAPR reduction performance as compared with the genetic algorithm-based TR method which has been known to have the best performance and obtains near-optimal PRT sets.
\end{abstract}

Keywords: Orthogonal frequency division multiplexing; Peak-to-average power ratio reduction; Tone reservation; Invasive weed optimization

\section{Introduction}

Orthogonal frequency division multiplexing (OFDM) has been attracting substantial attention due to its excellent performance under severe channel condition [1-4]. OFDM has been standardized in many wireless applications with high-speed data transmission such as terrestrial digital audio broadcasting and digital video broadcasting [2] and also has been implemented in wireless local area networks and wireless metropolitan area networks due to its robustness to multipath fading and bandwidth efficiency and other advantages. However, some challenging issues still remain unresolved in design of OFDM systems. One of the major problems is its sensitivity to peak-to-average power ratio (PAPR) of transmitted signals, and some schemes have been proposed to reduce the PAPR in OFDM systems

\footnotetext{
* Correspondence: yfahuang@mail.cyut.edu.tw

${ }^{3}$ Department of Information and Communication Engineering, Chaoyang University of Technology, Taichung 413, Taiwan

Full list of author information is available at the end of the article
}

$[3,4]$. High PAPR results in a way that an OFDM receiver's detection efficiency becomes very sensitive to nonlinear devices used in a signal processing loop, such as digitalto-analog converter (DAC) and high-power amplifier, which may severely impair performance due to induced spectral regrowth and detection efficiency degradation $[5,6]$. Therefore, it is very important to accurately identify PAPR distribution in OFDM systems to work out some effective measures to curb PAPR.

To reduce the PAPR of OFDM signals, numerous techniques have been proposed in the literature [3-17]. A comprehensive tutorial review of PAPR reduction techniques in OFDM systems is summarized in $[3,4]$. It is known that clipping [4] is the simplest method, but it degrades the bit error rate (BER) of the system and results in out-of-band noise and in-band distortion. Among all existing techniques of PAPR reduction, selective mapping (SLM) [7] and partial transmit sequence (PTS) [8-10] are very attractive due to their good PAPR reduction without the restriction on the 
number of subcarriers. However, in SLM technique, the requirement of multiple IFFT operations increases the implementation complexity. The PTS technique uses an iterative routine similar to the trial-and-error method in finding optimum phase factors that leads to lower PAPR. However, the PTS technique requires an exhaustive search over all combinations of allowed phase factors, whose complexity increases exponentially with the number of subblocks. Hence, it achieves considerable PAPR reduction without distortion, but the high computational complexity of multiple Fourier transforms is a problem in practical systems $[3,4,8-10]$. As a result, for all these search methods, either computational complexity is still high or PAPR reduction performance is not good enough.

To alleviate this problem, many PAPR reduction techniques have been proposed in the literature [3] for an overview. One of the classical and most popular techniques is known as tone reservation (TR) since no data is transmitted over the dedicated subcarriers [11-17]. In [18], a tone reservation algorithm has been developed where several subcarriers are set aside for PAPR reduction. Since the subcarriers are orthogonal, the additive signal on unused subcarriers causes no distortion to the data-bearing subcarriers. The TR technique attracted much attention for reducing PAPR for current and future OFDM standard systems because TR provides good PAPR reduction performance without BER performance degradation and signal distortion. In addition, the TR technique is simple and effective, and it causes no interference to the data signal. TR does not require the exchange of side information between transmitter and receiver. However, one of the disadvantages of TR is the increase in mean power of the transmitted signal because of corrective signal addition. Also, the computational complexity of the optimization algorithm is to calculate the optimized corrective tones which reduce the original signal's PAPR [11-15]. Therefore, practically, it is not realizable for a large number of peak reduction tone (PRT) set. Moreover, for these schemes, either the performance in PAPR reduction is suboptimal or the computational complexity is still high.

To tackle the complexity issue of TR, we formulate the sequence search of TR as a particular combinatorial optimization problem. To reduce the complexity for optimal peak reduction tone (OPRT) set, some stochastic search techniques [12-17] have recently been proposed because they could obtain the desirable PAPR reduction with a low computational complexity. Hence, the computational complexity is not significantly reduced. As a consequence, the key question is how to decrease the complexity while maintaining a PAPR reduction close to that of OPRT. In this paper, we take a fresh look at TR for PAPR reduction and propose solutions for both above-mentioned problems. In order to reduce computational complexity, some approaches have been proposed recently. Different evolutionary algorithms such as genetic algorithm (GA) [12] and cross entropy (CE) method [13] have been used for PAPR applications. Contrast to GA and $\mathrm{CE}$, invasive weed optimization (IWO) algorithm [19-24] and particle swarm optimization [25-28] are inspired from the phenomena of colonization of invasive weeds in nature which is based on the biology and ecology of weeds. In this paper, we propose a newly suboptimal PRT set selection scheme based on a modified IWO algorithm, which can efficiently reduce the PAPR of the OFDM signals. In the TR scheme, a small number of unused subcarriers called peak reduction carriers (PRCs) are reserved to reduce the PAPR, and the goal of the TR scheme is to find the optimal values of the PRCs that minimize the PAPR of the transmitted OFDM system. The proposed scheme can search the better combination of the initial PRT set to reduced PAPR. Simulation results show that the IWO-PRT optimization scheme can achieve superior PAPR reduction performance and at the same time requires far less computational complexity than the previous OPRT techniques. The rest of this paper is organized as follows. In Section 2, a typical OFDM system is given, the PAPR problem is formulated, and then PRT is explained. Then, IWO is proposed to search the optimal combination of PRT set for reduced PAPR in Section 3. Sections 4 and 5 discuss the simulation results and conclusions, respectively.

\section{OFDM system model and PAPR definition 2.1 OFDM system model}

In an OFDM system, a high-rate data stream is split into $N$ low-rate streams that are transmitted simultaneously by subcarriers, where $N$ is the number of subcarriers. Each of the subcarriers is independently modulated using phase-shift keying (PSK) or quadrature amplitude modulation (QAM). Inverse discrete Fourier transform (IDFT) generates the ready-to-transmit OFDM signal. For an input OFDM block $\mathbf{X}=\left[X_{0}, X_{1}, \ldots, X_{N-1}\right]^{T}$, each symbol in $\mathbf{X}$ modulates one subcarrier of $\left\{f_{0}, f_{1}, \ldots, f_{N-1}\right\}$. The $N$ subcarriers are orthogonal, i.e., $f_{n}=n \Delta f$, where $\Delta$ $f=\frac{1}{N T}$ and $T$ is the symbol period. The complex envelope of the discrete-time transmitted OFDM signal in one symbol period is given by [4]

$$
x_{n}=\frac{1}{\sqrt{N}} \sum_{i=0}^{N-1} X_{i} e^{j 2 \pi n i / L N}, n=0,1,2, \ldots, L N-1,
$$

$L$ is the oversampling factor, where $L=4$, which is enough to provide an accurate approximation of the PAPR [4] and $x_{n}$ is the $n$th signal component in OFDM output symbol. However, OFDM output symbols typically 
have a large dynamic envelope range due to the superposition process performed at the IFFT stage in the transmitter. PAPR is widely used to evaluate this variation of the output envelope. PAPR is an important factor in the design of both high-power amplifier (PA) and DAC and for generating error-free (or with minimum errors) transmitted OFDM symbols and also preventing the PA to work in nonlinearity region. It is shown in $[3,4]$ that choosing $L=4$ is sufficient to approximate the peak value of the continuous time OFDM signals.

\subsection{Peak-to-average power ratio}

The PAPR of the discrete-time baseband OFDM signal is defined as the ratio of the maximum peak power divided by the average power of the OFDM signal $[3,4,11]$, which is referred to as the PAPR, and is defined as

$$
\operatorname{PAPR}(x)=10 \log _{10} \frac{\max _{0 \leq n \leq L N-1}\left|x_{n}\right|^{2}}{E\left[\left|x_{n}\right|^{2}\right]},
$$

where $\max \left|x_{n}\right|^{2}$ is the maximum value of the OFDM signal power, and $E[\cdot]$ denotes the expected value operation. In principle, PAPR reduction techniques are concerned in the reduction of $\max \left|x_{n}\right|^{2}$. By applying the central limit theorem, assuming that the number of subchannels is sufficiently large, the time domain symbol is approximately a zero-mean complex that is Gaussian distributed and the power distribution becomes a central chi-square distribution with two degrees of freedom.

\subsection{Tone reservation scheme to reduce the PAPR}

In this paper, we consider the selection of the OPRT set for tone reservation [11-18,29-31] scheme to reduce the PAPR of an OFDM signal. TR scheme requires a sacrifice in data transmission efficiency because some subcarriers in an OFDM symbol should be reserved as peak reduction tones which are used only to reduce PAPR without carrying data. The size of PRT plays a critical role in TR scheme. To achieve lower PAPR, more subcarriers should be reserved as PRTs which reduces data transmission efficiency. TR technique is part in the reduction of the PAPR of an OFDM signal by reserving a few tones within the transmitted bandwidth and by assigning them the appropriate values $[18,29,30]$. In this paper, we formulate the optimal PRT set selection problem as a constrained combinatorial optimization and propose the application of the IWO method [19] to solve the problem.

In order to reduce the PAPR of OFDM signal using tone reservation scheme, some subcarriers are reserved as peak reduction tones set which are used to generate peak-canceling signal. In the TR-based OFDM scheme, peak reduction tones are reserved to generate PAPR reduction signals. These reserved tones do not carry any data information, and they are only used for reducing PAPR. Specifically, the peak-canceling signal $\mathbf{C}=\left[C_{0}, C_{1}, \ldots, C_{N-1}\right]^{T}$ generated by reserved PRT is added to the original time domain signal $\mathbf{X}=\left[X_{0}, X_{1}, \ldots, X_{N-1}\right]^{T}$ to reduce its PAPR. The PAPR-reduced signal can be expressed as [11-13]

$$
\mathbf{Y}=\mathbf{x}+\mathbf{c}=\Omega(\mathbf{X}+\mathbf{C})
$$

where $\mathbf{c}=\Omega \mathbf{C}$ is the peak-canceling signal in the time domain and $\mathbf{C}=\left[C_{0}, C_{1}, \ldots, C_{N-1}\right]^{T}$ is the avoid signal distortion; the data vector $\mathbf{X}$ and the peak reduction vector $\mathbf{C}$ lie in disjoint frequency domains, and $\mathbf{X}$ and $\mathbf{C}$ are not allowed to be non-zero on the same subcarriers, that is

$$
\mathbf{X}_{n}+\mathbf{C}_{n}=\left\{\begin{array}{l}
\mathbf{X}_{n}, n \in R^{C} \\
\mathbf{C}_{n}, n \in R
\end{array}\right.
$$

where $R=\left\{i_{0}, i_{1}, \ldots, i_{M-1}\right\}$ denotes the index set of the data-bearing tones (subcarriers), $R^{C}$ denotes the index set of the complementary set of $R$ in $N=\{0,1, \ldots, N-1\}$, and $M<N$ is the size of peak reduction tone set. With the TR scheme, the PAPR of the peak-reduced OFDM signal $\mathbf{Y}=\left[y_{0}, y_{1}, \ldots, y_{N-1}\right]^{T}$ is then redefined as [11-13]

$$
\operatorname{PAPR}(\mathbf{Y})=\frac{\max _{0 \leq n \leq N-1}\left|x_{n}+c_{n}\right|^{2}}{E\left[\left|x_{n}\right|^{2}\right]} .
$$

The PAPR reduction performance of the TR scheme mainly depends on the size of the PRT set, the maximum number of iteration, and the selection of PRTs. Thus $\mathbf{c}$ must be chosen to minimize the maximum of the peak-reduced OFDM signal $\mathbf{Y}$, i.e.,

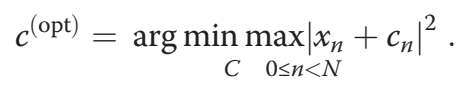

To compensate for this effect, we may simply follow an approach similar to [12], to obtain the PRT sets. In this paper, to obtain the optimum $c^{\text {(opt) }}$, Equation 7 can be reformulated as the following optimization problem:

$$
\begin{gathered}
\min _{C} E, \\
\text { subject to } E \geq 0, \\
\\
\left|x_{n}+c_{n}\right|^{2} \leq E,
\end{gathered}
$$

which is a quadratically constrained quadratic program (QCQP) problem [11] and $E$ is an optimization parameter. In order to find the optimal values of the PRCs, the TR optimization problem can be formulated as a QCQP problem. However, computing the exact solution to this QCQP problem is generally challenging. Therefore, the focus of the work is on the second approach. To reduce the complexity of QCQP, Tellado in [11] proposed a simple gradient algorithm to provide 
an approximate solution with reduced complexity. This iterative algorithm is used in this paper because of its low complexity. Although the optimum of a QCQP exists, the solution requires a high computational cost of $O\left(N_{s} N^{2} L\right)$, where $N_{s}$ is a complex number space. However, several low-complexity methods have been proposed $[11-13,18]$ and have achieved sufficient accurate suboptimal solutions. Although the optimum of a QCQP exists, the solution requires a high computational cost. To reduce the complexity of the QCQP, Tellado in $[11,18,30]$ proposed a simple gradient algorithm to iteratively approach $c$ and to update the vector as follows:

$$
\mathbf{c}^{(i+1)}=\mathbf{c}^{(i)}-\alpha_{i} \mathbf{p}\left[\left(\left(k-k_{i}\right)\right)_{n}\right]
$$

where $\alpha_{i}$ is a scaling factor relying on the maximum peak found at the $i$ th iteration, $\mathbf{p}=\left[p_{0}, p_{1}, \ldots, p_{N-1}\right]^{T}$ is called the time domain kernel, and $\mathbf{p}=\left[\left(\left(k-k_{i}\right)\right)_{N}\right]$ denotes a circular shift of $\mathbf{p}$ to the right by a value $k_{i}$, where $k_{i}$ is calculated by

$$
k_{i}=\underset{k}{\arg \max }\left|x_{k}+c_{k}^{(i)}\right|
$$

For ease of presentation, the time domain kernel is expressed as

$$
\mathbf{P}=\left[p_{0}, p_{1}, \ldots, p_{N-1}\right]^{T}=\Omega \mathrm{P}
$$

where $\mathbf{P}=\left[P_{0}, P_{1}, \ldots, P_{N-1}\right]^{T}, P_{n} \in\{0,1\}$ is called the frequency domain kernel whose elements are defined by

$$
P_{n}= \begin{cases}0, & n \in R^{C} \\ 1, & n \in R\end{cases}
$$

Then, the optimal frequency domain kernel $\mathbf{P}$ corresponds to the characteristic sequence of the PRT set $R$, and the maximum peak is always $\mathbf{p}$ because it is a $\{0,1\}$ sequence. The PAPR reduction performance depends on the time domain kernel $\mathbf{P}$, and the best performance can be achieved when the time domain kernel $\mathbf{p}$ is a discrete impulse because the maximum peak can be canceled without affecting other signal samples at each iteration. However, in order for the time domain kernel to be a discrete impulse, all the tones should be allocated to the PRT set. As the number of reserved tones becomes larger, the PAPR reduction performance is improved, but the data transmission rate decreases.

After I iterations of this algorithm, the peak-reduced OFDM signal is obtained:

$$
\mathbf{Y}=\mathbf{x}+\mathbf{c}^{(J)}=\mathbf{x}-\sum_{i=1}^{I} a_{i} \mathbf{p}\left[\left(\left(k-k_{i}\right)\right)_{N}\right]
$$

From Equations 8 to 12, it can be found that the PAPR reduction performance of the TR-based OFDM system depends on the selection of the time domain kernel, which is only a function of the PRT set. When there is a single discrete pulse, the best PAPR reduction performance can be obtained because the maximal peak at location can be canceled without distorting other signal samplings. However, it is impractical because a single discrete pulse will result in all tones being assigned to the PRT set. So we should select the time domain kernel such that it not only reduces the peak at location but also suppresses the other big values at location. To find the optimal PRT set, in mathematical form, we require solving the following combinatorial optimization problem:

$$
R^{*}=\arg \min _{R}\left\|\left[p_{1}, p_{2}, \ldots, p_{N-1}\right]^{T}\right\|_{\infty}
$$

where $\|\cdot\|_{j}$ denotes the $j$-norm and $\infty$-norm refers to the maximum values. It is known that this problem is NP-hard because the time domain kernel $\mathbf{p}$ must be optimized over all possible discrete sets $R$ [30]. which requires an exhaustive search of all combination of possible PRT set, i.e. possible combination numbers of PRT set are searched, where denotes the binomial coefficient. It is a non-deterministic polynomial time (NP)-hard problem and cannot be solved for the number of tones envisaged in practical systems. In $[18,30]$, the consecutive PRT set, the equally spaced PRT set, and the random PRT set optimization were proposed as the candidates of PRT set. Although the consecutive PRT set and the equally spaced PRT set are the simplest selections of PRT set, their PAPR reduction performance are inferior to that of the random PRT set optimization. However, the random PRT set optimization requires a larger PRT set sampling, and the associated complexity limits the application of such a technique. A variance minimization method in [31] is developed to solve the NP-hard problem, and it is just a modified version of the random PRT set optimization, which also has the drawback of high computational cost. In [15], a cross entropy method was proposed to solve the problem. It obtains better results than the existing selection methods, but it requires a larger population or sampling size. These limitations of the existing methods motivate us to find an efficient method to obtain a nearly optimal PRT set. As mentioned before, we propose an IWO-based PRT set selection method for the purpose of having a very low computational complexity.

\subsection{PRT position search based on IWO algorithm}

A detailed description of the IWO used for searching the nearly optimal PRT set positions is described as follows. An initial population of plants (weed) is randomly generated. Each plant sequence is a vector of length $N$, and each element of the vector is a binary zero or 
one depending on the existence of a PRT at that position (one denotes existence and zero denotes non-existence). The number of the PRT in each binary vector is $M<N$.

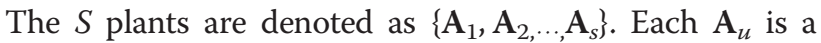
binary vector of length. For each plant $\mathrm{A}_{u}$, the PRT set $R_{u}$ is the collection of the locations whose elements are one. Then, the frequency domain kernel corresponding to the PRT set is obtained using Equation 11, and the time domain kernel $P_{u}=\left[p_{0}^{u}, p_{1}^{u}, \cdots, p_{N-1}^{u}\right]$ is obtained using Equation 10. The merit (secondary peak) of the sequence is defined as [12]

$$
m\left(A_{u}\right)=\left\|\left[p_{1}^{u}, p_{2}^{u}, \ldots, p_{N-1}\right]^{T}\right\|_{\infty} .
$$

The $T$ sequences (called elite sequences) with the lowest merits are maintained for the next population generation. The best merit of the sequences is defined as

$$
\hat{m}=\min _{1 \leq u \leq N} m\left(A_{u}\right) .
$$

\section{Minimizing PAPR using modified invasive weed optimization-based PRT set}

\subsection{IWO algorithm}

In recent years, Mehrabian and Lucas proposed a novel population-based stochastic search method called IWO mechanism to achieve global optimization [19]. IWO is a numerical stochastic optimization technique inspired by the colonization of weeds. As a result of investigation, weeds have shown to be very robust and adaptive to changes in the environment, where capturing their properties leads to a very powerful optimization technique [19-25]. Most importantly, the IWO method has shown its robustness in practice. Hence, by applying the IWO algorithm into the PRT scheme, it could provide a way to reduce the PAPR of OFDM transmitted signal. The performance evaluation of the proposed scheme for PAPR reduction and computation complexity is given in this paper. We show that not only the PAPR is reduced but also the complexity and processing time is decreased. However, to our knowledge, IWO [19-24] has not yet been used for the same purpose till now. In this work, we extend the classical IWO algorithm for handling PAPR problems. We propose a hybrid schedule to decrease the variance of the seed populations in IWO and also use the concept of particle swarm optimization (PSO) [25-28] for choosing the best maximum number of population members that will survive to the next generation.

IWO, first designed and developed by Mehrabian and Lucas [19], is a relatively novel numerical stochastic optimization algorithm inspired from colonization of invasive weeds. A weed is any plant growing where it is not wanted; any tree, vine, shrub, or herb may qualify as a weed in any specified geographical area, depending on the situation. Weeds have shown a very robust and adaptive nature that renders them undesirable plants in agriculture. In a $D$-dimensional search space, a weed which represents a potential solution of the objective function is represented as $\mathbf{p}=\left(p_{1}, p_{2}, \ldots, p_{N-1}\right)$. Firstly, $p$ weeds, called a population of plants, are initialized with random growth position, and then each weed produces seeds depending on its fitness and the colony's lowest fitness and highest fitness to simulate the natural survival of the fittest process. The number of seeds each plant produces increases linearly from minimum possible seed production to its maximum; the generated seeds are being distribution randomly in the search area by normal distribution with mean the equal to zero and a variance parameter decreasing over the number of iteration. By setting the mean parameter equal to zero, the seeds are distributed randomly such that they locate near the parent plant, and by decreasing the variance over time, the fitter plants are grouped together and inappropriate plants are eliminated over time. The general scheme for the IWO algorithm is shown in Algorithm 1, which consists of four main procedures: initialization, reproduction, spatial dispersal, and competitive exclusion operator, respectively. [20-24]:

\section{Algorithm 1 Invasive weed optimization algorithm 1 \\ 1. Initialize population space( ); select an initial population of $N$ candidate solution, Evaluate fitness}

2. while termination criteria are not satisfied do

\section{Reproduction ()}

4. Spatial dispersal ()

\section{Competitive exclusion ()}

end while

The produced seeds in this step are being dispread over the search space by normally distributed random numbers with the mean equal to the location of the producing plants and varying standard deviations. The standard deviation (SD) at the present time step can be expressed by

$$
p_{\text {iter }}=\left[\frac{\left(\text { iter }_{\mathrm{MAX}}-\text { iter }^{n}\right.}{\left(\text { iter }_{\mathrm{MAX}}\right)^{n}}\right]\left(p_{\text {initial }}-p_{\text {final }}\right)+p_{\text {final }},
$$

to generate a new feasible population by randomly adding or removing PRTs, where iter ${ }_{\mathrm{MAX}}$ is the maximum number of iterations, $w_{\text {iter }}$ is the $\mathrm{SD}$ at the present time step, and $n$ is the nonlinear modulation index. This alteration ensures that the probability of dropping a seed in a distant area decreases non-linearly at each time step, 
which results in the grouping of fitter plants and the elimination of inappropriate plants. Figure 1 shows the standard deviation over the course of a run with 200 iterations and different modulation indexes.

\subsection{Particle swarm optimization}

Kennedy [26] invented PSO as one of the most powerful members of the class of stochastic search techniques in 2001. They are originally defined to solve NP-complete problems. However, two optimization techniques are compared in this section. One advantage of PSO [27] over the GA is its algorithmic simplicity. A GA comprises parameters of its major operators which are crossover, mutation, and elitism. The parameters are population size, probability of mutation, probability of crossover, and selection. However, PSO has one simple operator, velocity calculation. The benefit of having a small number of operators is the reduction of computation and the elimination of the need to select the best operator for a given optimization. Another difference between the PSO and GA is the ability to control convergence. Mutation and crossover rates can subtly affect the convergence of the GA, but not as effectively as the inertial weight. Fogle [27] indicated that the decrease of inertial weight significantly increases the swarm's convergence. This type of control allows its use to determine the rate of convergence, and the final level of stagnation is ultimately achieved. Stagnation occurs in the GA when eventually all the individuals possess primarily the same genetic code. In that case, the gene pool is so homogeneous that crossover has little or no effect, and each successive generation is the same as the first.

In this context, the population is called a swarm and the individuals are called particles. Resembling the social behavior of a swarm of bees to search the location with the most flowers in a field, the optimization procedure of PSO is based on a population of particles which fly in the solution space with velocity dynamically adjusted

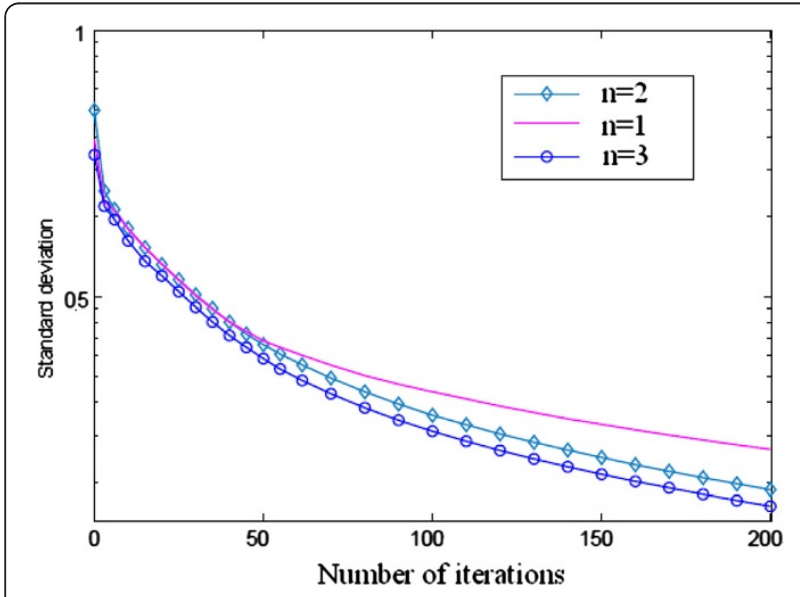

Figure 1 Standard deviation over the course of the run. according to its own flying experience and the flying experience of the best among the swarm. During the PSO process, each potential solution is represented as a particle with a position vector and a moving velocity represented as $\mathbf{x}$ and $\mathbf{v}$, respectively. Thus for a $K$-dimensional optimization, the position and velocity of the $j$ th particle can be represented as $\mathbf{x}_{j}=\left(\mathbf{x}_{j, 1}, \mathbf{x}_{j, 2}, \ldots, \mathbf{x}_{j, K}\right)$ and $\mathbf{v}_{j}=\left(\mathbf{v}_{j, 1}, \mathbf{v}_{j, 2}, \ldots, \mathbf{v}_{j, K}\right)$. Like a GA, the PSO also begins by generating a population of particles at random. At each time step, an associated value for each particle is evaluated in accordance with a function called the fitness function which is critically defined and configured from a consideration of the search objective. The value normally called the fitness indicates the goodness of the solution. The position of the individual best fitness which the $i$ th particle has been achieved so far; that of the highest fitness which has been obtained among all the particles in the population so far are known as the personal best (denoted as $\mathbf{x}_{j}^{\text {best }}$ ) and the global best (denoted as $\mathbf{x}^{\text {best }}$ ), respectively, and both are stored to generate the new velocity of $j$ th particle. During the process, each particle adjusts its velocity according to its own experience, and the position of the best of all particles moves toward the best solution.

In the meantime, a condition is also set during the following step which controls the algorithm when it stops by either setting it to obtain an acceptable target solution or to run for a set maximum number of search iterations $\mathbf{G}$. If the algorithm does not stop, after a time step, $\Delta t$, the new velocity $\mathbf{v}_{i}(t+\Delta t)$ for particle $i$ is updated by

$$
\begin{aligned}
\mathbf{v}_{i}(t+\Delta t)= & w * \mathbf{v}_{i}(t)^{i}+c_{1} * \operatorname{rand}() *\left(x_{i}^{\text {best }}(t)-x_{i}(t)\right) \\
& +c_{2} * \operatorname{rand}() *\left(x^{\text {best }}(t)-x_{i}(t)\right)
\end{aligned}
$$

where $\mathbf{v}_{i}(t)$ is the old velocity of particle $i$ at time $t$. Significantly, in the real implementation of a PSO, time $t$ and $t+\Delta t$ generally represent the current and next iterations, respectively, so $\Delta t$ in Equations 17 and 18 is always omitted in most literature. This equation clearly indicates that the new velocity is related to the old velocity weighted by $w(t)$ and is also associated with the position of the particle itself and of the global best one by factors $c_{1}$ and $c_{2}$, respectively. The parameters $c_{1}$ and $c_{2}$ which are set to constant value are therefore known as the cognitive and social rates, respectively, since they represent the weighting of the acceleration terms that pull the individual particle toward the personal best and global best positions. The inertia weigh $w$ in Equation 17 is employed to manipulate the impact of the previous history of velocities on the current velocity. Conversely, in the later stages, convergence toward the global optima should be enhanced to find the optimum solution 
efficiently. Therefore, $w$ resolves the trade-off between the global and local exploration ability of the swarm. Ratnaweera et al. [28] introduced time-varying acceleration coefficient, which reduces the cognitive component and increases the social component of acceleration coefficient. For the purpose of intending to simulate the slight unpredictable component of the natural swarm behavior, two random functions rand() are applied to independently provide uniformly distributed numbers in the range $[0,1]$ to stochastically vary the relative pull of the personal and global best particles. Based on the updated velocities, new position for particle $j$ is computed according the following equation:

$$
x_{j}(t+1)=x_{j}(t)+v_{j}(t+1) .
$$

The populations of particles are then moved according to the new velocities and locations calculated by Equations 17 and 18 and tend to cluster together from different directions. Thus, the evaluation of each associate fitness values of the new population of particles begins again. The algorithm runs through these processes iteratively until it stops. In Equation 17, the inertial weight factor provides the necessary diversity to the swarm by changing the momentum of particles and hence avoids the stagnation of particles at the local optima. Usually, it needs to define a maximum velocity for each modulus of velocity vector, which is often set as the upper limit of each modulus of position vector. This helps to control the unnecessary excessive roaming of particles outside the predefined search space. The role of $w$ is considered critical for the convergence behavior of PSO. Given a user-specified maximum weight $w_{\max }$ and a minimum weight $w_{\min }$, the inertial weight $w$ is updated as the following monotonically decreasing function of the iteration $g$ :

$$
w_{g+1}=w_{g}+\frac{\left(w_{\min }-w_{\max }\right)}{G_{\max }},
$$

where $G_{\max }$ is the predefined maximum number of iterations and $g$ is the iteration number. It has been demonstrated that 0.9 for $w_{\max }$ and 0.4 for $w_{\min }$ can greatly improve the performance of PSO.

\subsection{The hybrid IWO/PSO algorithm}

IWO and PSO algorithms are both derived from biologic behavior. Although these algorithms go through similar evolution processes, including selection, reproduction, recombination, and mutation, they are two approaches with different propagation methods. In the hybrid algorithm, the IWO algorithm plays the role of guiding the evolution and the PSO algorithm works as an assistant. The interaction between the dispersion method of the IWO algorithm and the velocity of the PSO algorithm controls the balance between local exploitation and global exploration in the problem space. The process of the hybrid algorithm is formulated in detail as follows:

Step 1. Seeds are produced. First, the variables that need to be optimized should be determined. Each variable is initiated in its solution space. The solution of each variable is a particle, and a set of particles for the variables form a seed; i.e., each seed is an initial solution of the problem. A number of seeds constitute a colony.

Step 2. Seeds grow into plants. Each seed is assessed according to its fitness value, which is obtained from the fitness function defined to represent the goodness of the solution. After the fitness value is assigned to the corresponding seed, the seed grows into a flowering plant, i.e., a weed.

Step 3. Each plant finds its position in the colony. A group of plants are ranked based on their fitness values. The $i$ th plant denotes the $i$ th initial position, which is $p_{\text {best }}$. The most satisfactory fitness value is the best position of the colony $g_{\text {best }}$.

Step 4. The velocities and positions of all plants are modified. The next velocities and positions of all plants are renewed based on Equations 17 and 18:

$$
\begin{aligned}
\mathrm{v}_{i}(t+\Delta t)= & w \times \mathrm{v}_{i}(t)^{i}+c_{1} \times \operatorname{rand}() \times\left(x_{i}^{\text {best }}(t)-x_{i}(t)\right) \\
& +c_{2} \times \operatorname{rand}() \times\left(x^{\text {best }}(t)-x_{i}(t)\right), \\
& \text { and } x_{i}(t+1)=x_{i}(t)+v_{i}(t+1) .
\end{aligned}
$$

where $\mathrm{t}$ is the iterative time, $v i$ and $x i$ are the velocity and position of the $i$ th plant, respectively, $w$ is the inertia weight, and $c 1$ and $c 2$ are the learning factors.

Step 5. The flowering plants produce new seeds. The number of seeds produced by each plant depends on its fitness value ranking and decreases from the maximum possible seed production $\Theta_{\max }$ to the minimum $\Theta_{\text {min }}$.

Step 6. The seeds are dispread over the solution space by normally distributing random numbers with mean equal to the locations of the producing seeds and varying standard deviations. The SD at the present time step can be expressed as Equations 16 and 20:

$$
\begin{aligned}
& p_{\text {iter }}=\left[\frac{\left(\text { iter }_{\text {MAX }} \text {-iter }\right)^{n}}{\left(\text { iter }_{\text {MAX }}\right)^{n}}\right]\left(p_{\text {initial }}-p_{\text {final }}\right)+p_{\text {final }} \\
& x_{i}(t+1)=x_{i}(t+1)+\text { rand } \times p_{\text {iter }}
\end{aligned}
$$

where iter $_{\max }$ is the maximum number of iterations, $p_{\text {initial }}$ and $p_{\text {final }}$ are the initial and final standard deviations, respectively, and $n$ is the non-linear modulation index.

Step 7. The new seeds are ranked with their parents based on their fitness values and find their positions in the colony. The seeds with a higher ranking grow into flowering plants, and those with a lower 
ranking in the colony are eliminated to reach the maximum number of plants in the colony.

Step 8. The surviving seeds can produce new seeds based on their ranking in the colony. The process is repeated until either the maximum number of iterations is reached or the fitness criterion is met.

Therefore the proposed IWO-PSO-based PRT position search algorithm can be summarized in Algorithm 2:

\section{Algorithm 2 Invasive weed optimization algorithm 2}

Step 1. First of all, the parameters (variables) that need to be optimized should be selected. Then, for each of these variables in the $D$-dimensional solution space, a maximum and a minimum value should be assigned (defining the solution space). Population size, acceleration coefficients, inertia weights, initial SD, number of candidate PRT set $W$, and maximal iteration number $\mathbf{I}$ are set.

Step 2. An initially feasible population of size is randomly generated, and the PRT set for each sequence is found. Each initial seed grows to a flowering plant. That is, the fitness function, defined to represent the goodness of the solution, returns a fitness value for each seed. After assigning the fitness value to the corresponding seed, it is called a plant. An initially feasible population of size is randomly generated, and the PRT set for each sequence is found. The frequency domain kernel using Equation 11 and the time domain kernel using Equation 10 for each sequence are calculated.

Step 3. The merits (secondary peaks) are calculated using Equation 14, elite sequences are selected with the lowest merits, and the best merit using Equation 15 and the corresponding PRT set are found.

Step 4. The produced seeds in this step are being dispread over the search space by normally distributed random numbers with the mean equal to the location of the producing plants and varying standard deviations. The seeds are dispread over the solution space by normally distributing random numbers with the mean equal to the locations of the producing seeds and varying standard deviations. The SD at the present time step can be expressed as Equations 16 and 20:

$$
\begin{aligned}
& p_{\text {iter }}=\left[\frac{\left(\text { iter }_{\mathrm{MAX}}-\text { iter }^{n}\right.}{\left(\text { iter }_{\mathrm{MAX}}\right)^{n}}\right]\left(p_{\text {initial }}-p_{\text {final }}\right)+p_{\text {final }} \\
& x_{i}(t+1)=x_{i}(t+1)+\operatorname{rand} \times p_{\text {iter }}
\end{aligned}
$$

where iter $_{\max }$ is the maximum number of iterations, $p_{\text {initial }}$ and $p_{\text {final }}$ are the initial and final standard deviations, respectively, and $n$ is the non-linear modulation index. This alteration ensures that the probability of dropping a seed in a distant area decreases non-linearly at each time step, which results in the grouping of fitter plants and the elimination of inappropriate plants.

Step 5. The merits and the best merit of the new population are evaluated. If the best merit of the new population is smaller than that of the previous generation, then the best merit and the corresponding PRT set are updated. Otherwise, the previous best merit and the corresponding PRT set unchanged are retained.

Step 6. The worst sequences with the highest merits in the current generation by the elite sequences from the previous generation are replaced, and elite sequences are reselected.

Step 7. The new seeds are ranked with their parents based on their cost values, and their positions in the colony are found. The seeds with a lower ranking grow into flowering plants, and those with a lower ranking in the colony are eliminated to reach the maximum number of plants in the colony.

Step 8. If the maximal iteration number is achieved, there is an output for the PRT set and the corresponding secondary peak, and the algorithm is terminated; Steps 3 and 4 are repeated until a stopping criterion is satisfied. 


\section{Results and discussions}

Let us now proceed to the application of the IWO-based tone reservation method in OFDM PAPR reduction. In the simulations, we consider an OFDM system with $N=64,128$ and 256 subcarriers; data symbols are modulated using the QPSK and16-QAM constellation, and the number of reserved PRT set is at $W=8$ and 16 , respectively. In order to generate the complementary cumulative distribution function $(\mathrm{CCDF})=\left(\mathrm{PAPR}>\mathrm{PAPR}_{0}\right)$ of the PAPR, $10^{-4}$ OFDM blocks are generated randomly, where the transmitted signal is oversampled by a factor of $L=4$.

Figure 2 compares the average PAPR reduction performance of the IWO-TR, CE-TR, and GA-TR with the PRT set for the same iteration numbers. Figure 2 shows that the average PAPR reduction performance of the IWO-TR algorithm is better than those of CE-TR and GA-TR with different modulations. When the iteration number equals 120 , the IWO-TR algorithm converges to $5.8 \mathrm{~dB}$ PAPR with 16-QAM. Although the GA-TR algorithm is almost simple, its convergence speed is the slowest among the three methods. When the iteration number is 100 , its average PAPR is approximately $6.3 \mathrm{~dB}$, which is $0.52 \mathrm{~dB}$ larger than the IWO-TR algorithm in 120 iterations. The CE-TR algorithm converges to $6 \mathrm{~dB}$ PAPR in 120 iterations, however, which is approximately $0.2 \mathrm{~dB}$ larger than IWO-TR algorithm in the same iterations. Note that the PAPR performance of the IWO algorithms and the CE method is close to optimal. This shows that adding flexibility to the choice of the number of iterations and modulations can improve the convergence speed of the algorithm. Finally, this paper shows the trade-off between the number of iterations and modulation for the PAPR reduction.

The distribution of the PAPR bears stochastic characteristics in a practical OFDM system, which often can be

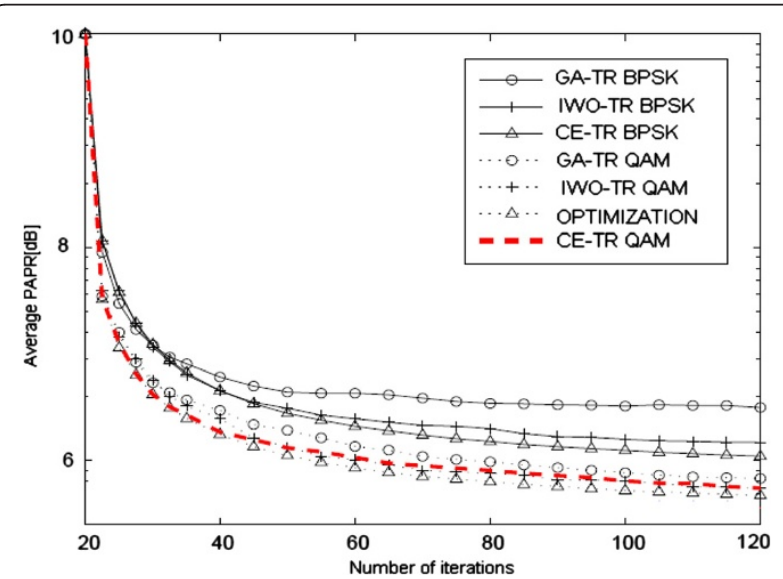

Figure 2 Relationship of PAPR reduction with the number of iterations and modulation for different techniques. expressed in terms of CCDF. The CCDF itself can be used to estimate the bounds for the minimum number of redundancy bits required to identify the PAPR sequences and evaluate the performance of any PAPR reduction schemes. Therefore, we mainly discuss the shapes of CCDF obtained by different searching methods for the combination of phase factors. In this section, the system performance with applied proposed IWO-based PRT scheme is evaluated based on the PAPR CCDF [4] by computer simulation. The cumulative distribution function (CDF) of the amplitude of a sampling signal is computed as $\mathrm{CDF}=1-\exp \left(\mathrm{PAPR}_{0}\right)$, and the CCDF $[2,3]$ could be defined as

$$
\begin{aligned}
\mathrm{CCDF} & =P_{r}\left(\mathrm{PAPR}>\mathrm{PAPR}_{0}\right) \\
& =1-P_{r}\left(\mathrm{PAPR} \leq \mathrm{PAPR}_{0}\right)=1-\left(1-\exp \left(-\mathrm{PAPR}_{0}\right)\right)^{N} .
\end{aligned}
$$

In Figure 3, some results of the CCDF of the PAPR are simulated for the OFDM system with 64 subcarriers and 16-QAM modulation, the number of the reserved tones $W=8$ employing random partition and the numbers of iterations are used for PAPR reduction. In Figure 3, we select the GA mentioned, in which the trial of PRT set combination is randomly generated to compare the performance of PAPR reduction with that of the IWO method. It is evident that IWO can provide consistent improvements of PAPR reduction than GA with some significant gains in the low PAPR range. In Figure 3, the CCDFs for OFDM without PAPR reduction and for optimal PRT with high enumeration are included. When $C C D F=\left(P A P R>P_{P A R}\right)=10^{-4}$, the $\mathrm{PAPR}_{0}$ of the original OFDM, GA-, CE- and IWO-TR are 12, 7.2, 6.5 , and $6.30 \mathrm{~dB}$ with iterations 50, respectively. However, when $P_{r}\left(\mathrm{PAPR}>\mathrm{PAPR}_{0}\right)=10^{-4}$, the PAPR performance of our proposed IWO-based PRT scheme with 30 iteration not only is almost the same as that of the GA-based PTS scheme with 50 iteration but is also having a much lower computational complexity. In general, in order to obtain an optimal PAPR, a search for the number of iterations must be accomplished.

As shown in Figure 4, as the maximum number of iterations is increased, the CCDF of the PAPR has a better performance. The comparison is carried out under the same conditions of initial population of candidate solutions and number of iterations. Figure 4 compares the PAPR reduction performance of the proposed IWO algorithm based PRT sets, GA, and CE algorithm for the same iterations. The same maximum iteration number is set to 50 for GA-TR, CE-TR, and IWO-TR schemes. When $P_{r}\left(\mathrm{PAPR}>\mathrm{PAPR}_{0}\right)=10^{-4}$, the PAPR of the original OFDM is $12.2 \mathrm{~dB}$. Using the GA-TR algorithm, IWO-TR algorithm, and CE-TR algorithm with 128 subcarriers, the PAPRs are approximately reduced 


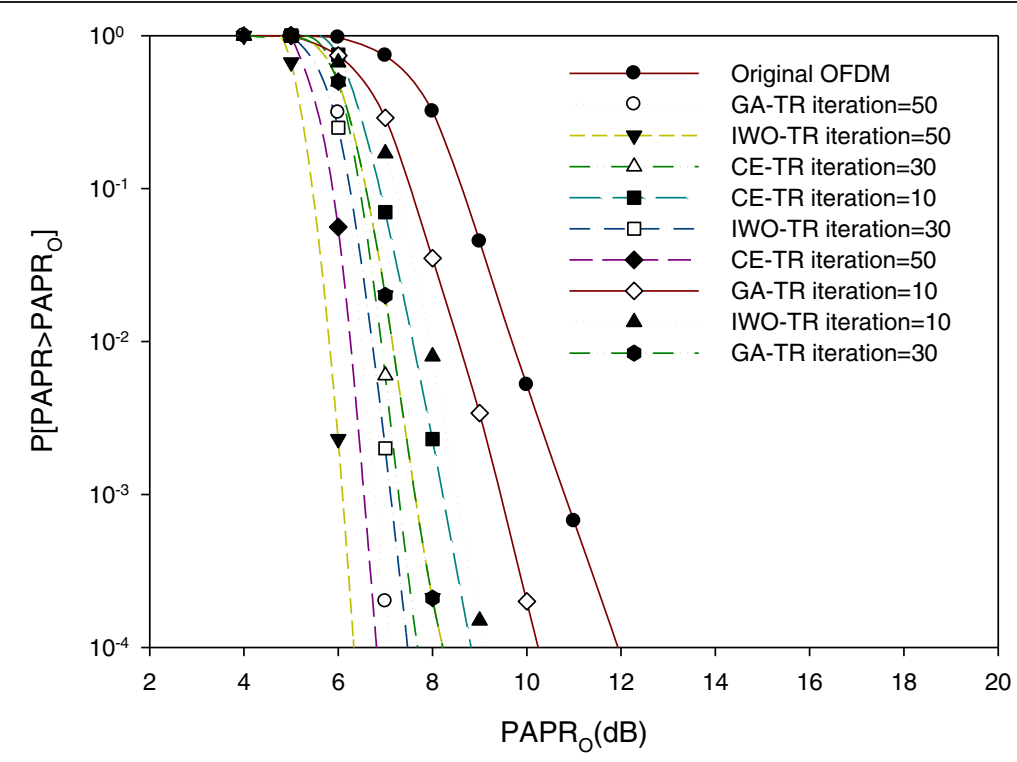

Figure 3 PAPR CCDF comparison of different maximum numbers of iterations of the IWO method for $N=64, W=8$.

to $7.23,7.1$, and $6.56 \mathrm{~dB}$, respectively. From Figure 3, it can be seen that apart from the PAPR by OPTS in [11], the PAPR reduction performance of the IWO-PTS is the best among all of the methods for the same or almost the same search complexity.

Figure 5 shows a comparison of the CCDF of PAPR for the conventional OFDM, the GA-PRT, the CE-PRT, and the proposed IWO-PRT set in an OFDM system with $N=256$ subcarriers. Figure 5 illustrates that the proposed IWO scheme with iteration $=30$ outperforms the GA method with iteration $=50$ and can achieve almost the same PAPR reduction performance as both the conventional PTS and SD-PTS schemes. Since the computation complexity reduction ratio increases as the number of subcarriers, the proposed scheme becomes more suitable for the high data rate OFDM system. Next, a fair comparison of the performance-complexity trade-offs for the different stochastic PTS searching strategies with $N$ and $W$, respectively, is provided in Figures 3,4 , and 5 , where the average PAPR reduction

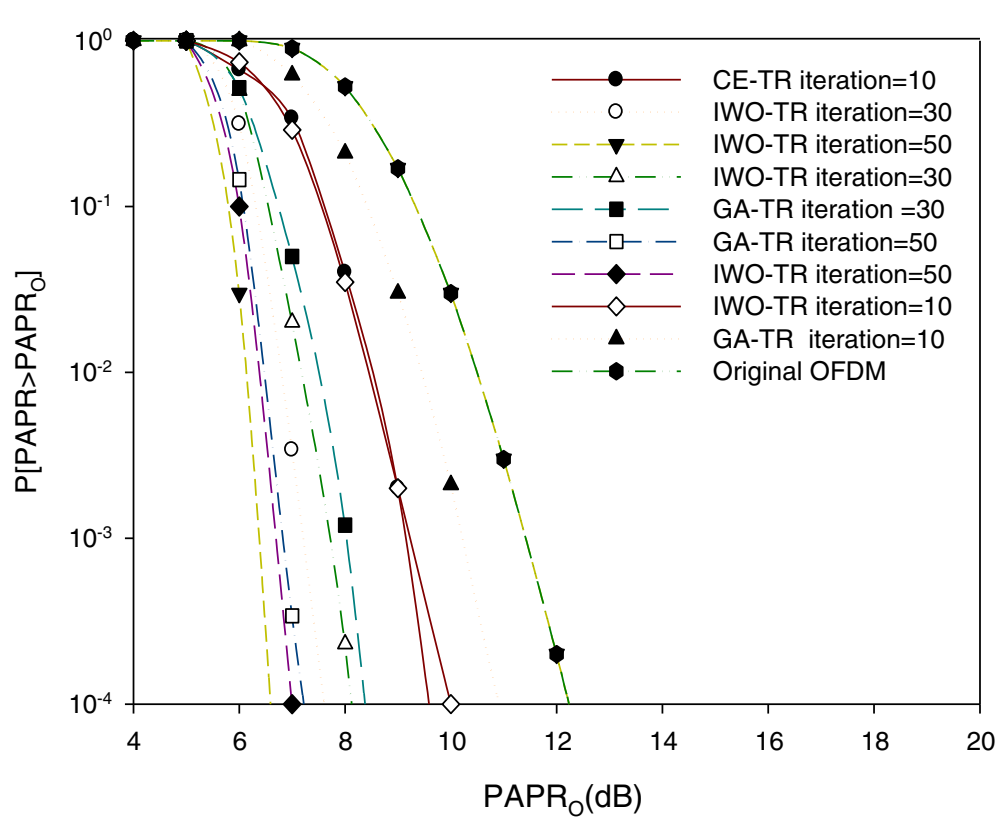

Figure 4 PAPR CCDF comparison of different maximum numbers of iterations of the IWO method for $N=128$ and $W=16$. 


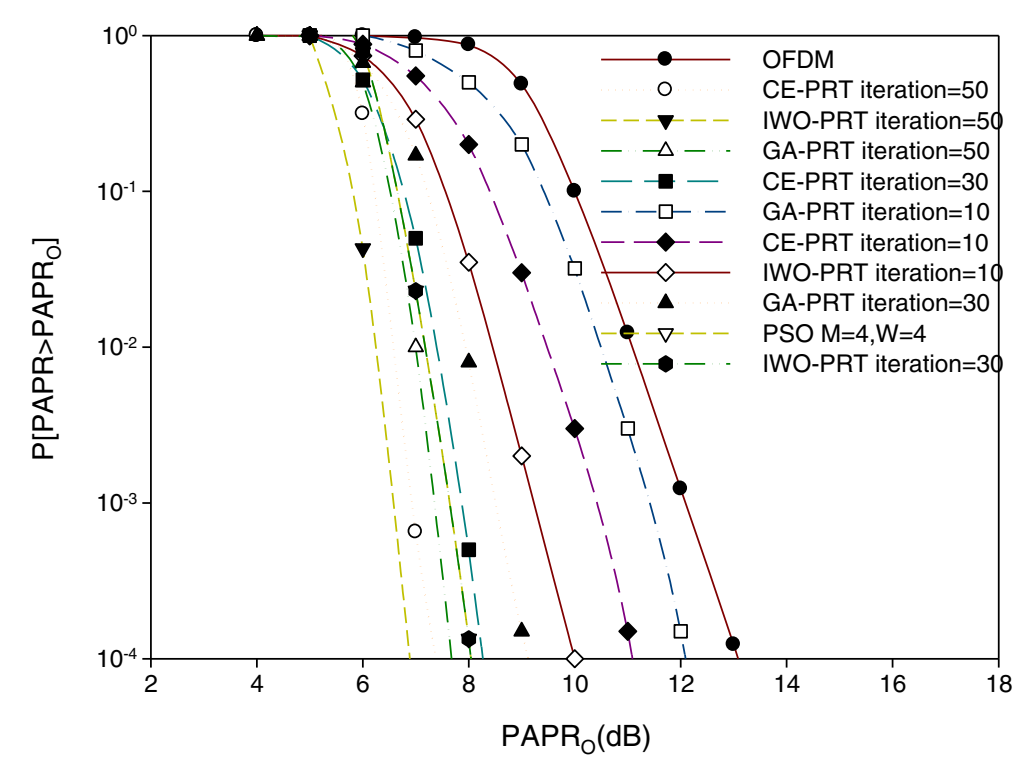

Figure 5 PAPR CCDF comparison of different maximum numbers of iterations of the IWO method for $N=256$ and $W=32$.

is the function of the number of sample. It can be seen that (1) it is beneficial to select more samples so that the average PAPR reduction performance can be improved, and (2) the application of the IWO method to solve the optimum PRT set problem yields an enhanced trade-off in the low average PAPR range of approximately 6.72 and $8.76 \mathrm{~dB}$ for $W=$ and $W=16$, respectively. Therefore, in this case, we proposed an improved TR scheme, which is based on the TR algorithm, to reduce the PAPR of OFDM system. In summary, based on the proposed IWO searching for the optimal combination of PRT set, we conclude that (1) the proposed IWO method can reduce the computational complexity as shown in Figures 3, 4, and 5, and (2) the proposed IWO method can obtain almost the same PAPR reduction as that of optimal PRT sequences illustrated in Figure 2.

In Figure 6, we compare the PAPR reduction performance of the IWO-PRT with different modulations, with the same size of population $P=30$, and with the same maximum iteration number $I=30$ for $W=16$. When sampling $=3,000$, the average PAPR of the GA-PRT with QPSK and 16-QAM are 8.12 and $7 \mathrm{~dB}$, respectively. The average PAPR by the IWO-PRT with QPSK and 16-QAM are 7.2 and $4.85 \mathrm{~dB}$, respectively. From Figure 6, it can be discovered that the difference of the PAPR between QPSK and 16-QAM is important. Little performance improvement can be obtained by different modulation schemes.

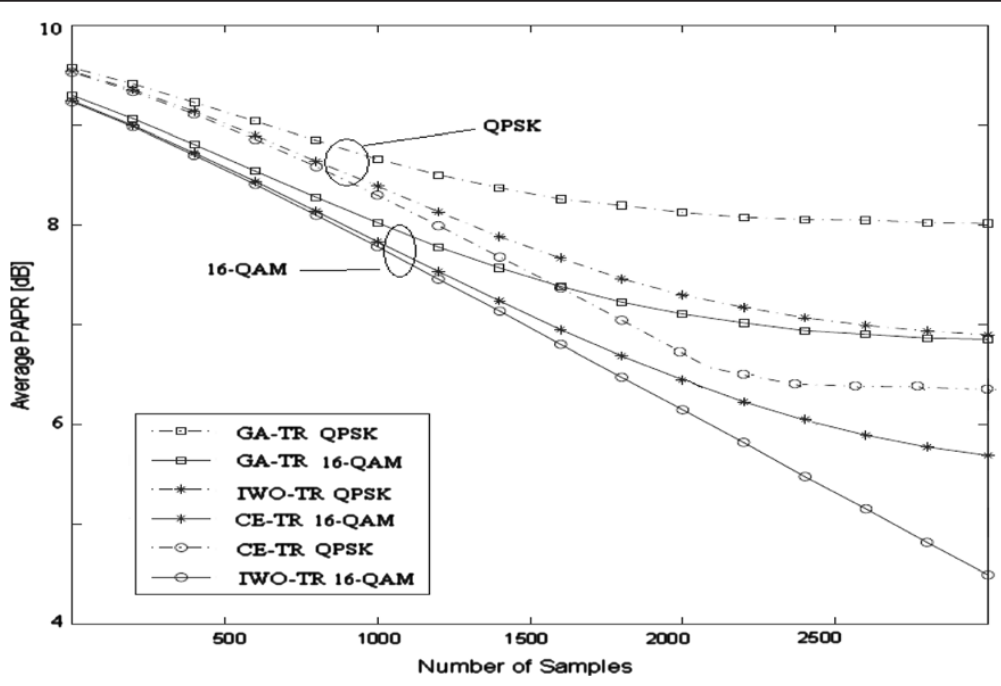

Figure 6 Average PAPR reduction comparison of the proposed scheme with different modulation QPSK and QAM. 


\section{Conclusions}

This paper presents an IWO-based method used to search a nearly optimal PRT set in the TR method for the improvement of peak-to-average power ratio performance of the OFDM system. The aim of this paper is to review the conventional PAPR reduction schemes and the various different evolutionary algorithms based on conventional PAPR reduction schemes to achieve a low computational complexity. The simulations have been conducted and proven that IWO is an effective method to yield an enhanced trade-off between PAPR reduction and complexity. Since the computational complexity reduction ratio increases as the number of subcarriers increases, the proposed scheme becomes more suitable for the high data rate OFDM systems.

\section{Competing interests}

The authors declare that they have no competing interests.

\section{Authors' information}

HLH received his M.S. degree in electrical engineering from the University of Detroit Mercy, Michigan, USA in 1994 and his Ph.D. degree in electrical engineering from National Chung Cheng University, Chia-Yi, Taiwan in 2007. From 1995 to 2006, he was a lecturer with the Department of Electrical Engineering, Chienkuo Technology University, Taiwan. Since 2007, he was an associate professor of the Department of Electrical Engineering, Chienkuo Technology University, Taiwan. His current research interests are in wireless communications, detection of spread-spectrum signal, wireless sensor networks, evolutionary computation, and intelligent systems. HLH serves as an associate editor for the Telecommunication Systems. YFH is a professor at the Department of Information and Communication Engineering, in Chaoyang University of Technology.

\section{Acknowledgements}

The works of $\mathrm{H}-\mathrm{L}$ Hung and $\mathrm{Y}-\mathrm{F}$ Huang were supported in part by the National Science Council (NSC) of Taiwan under grant NSC 101-2221-E-324-024.

\section{Author details}

${ }^{1}$ Department of Electrical Engineering, Chienkuo Technology University, Changhua City 500, Taiwan. ${ }^{2}$ Metal Industries Research \& Development Centre, Taichung 40768, Taiwan. ${ }^{3}$ Department of Information and Communication Engineering, Chaoyang University of Technology, Taichung 413, Taiwan.

Received: 18 May 2013 Accepted: 26 September 2013 Published: 17 October 2013

\section{References}

1. R van Nee, R Prasad, OFDM for Wireless Multimedia Communications (Artech House, Boston, 2000)

2. Digital Video Broadcasting (DVB), Framing Structure, Channel Coding and Modulation for Digital Terrestrial Television (ETSI, Sophia-Antipolis, 2009). ETSI EN 300744 V1.6.1

3. SH Han, JH Lee, An overview of peak-to-average power ration reduction techniques for multicarrier transmission. IEEE Wireless Commun. 12(2), 56-65 (2005)

4. T Jiang, W Yiyan, An overview: peak-to-average power ratio reduction techniques for OFDMsignals. IEEE Trans. on Broadcasting 54(2), 257-268 (2008)

5. S Litsyn, G Wunder, Generalized bounds on the crest-factor distribution of OFDM signals with applications to code design. IEEE Trans. Inform. Theory 52, 992-1006 (2006)

6. T Jiang, W Yao, P Guo, Y Song, D Qu, Two novel nonlinear companding schemes with iterative receiver to reduce PAPR in multicarrier modulation systems. IEEE Trans. Broadcast. 52(2), 268-273 (2006)

7. RW Baml, RFH Fisher, JB Huber, Reducing the peak-to-average power ratio of multicarrier modulation by selected mapping. Electron. Lett. 32(22), 2056-2057 (1996)
8. Y Wang, W Chen, C Tellambura, A PAPR reduction method based on artificial bee colony algorithm for OFDM Signals. IEEE Trans. on Wireless Commun. 9(10), 2994-2999 (2010)

9. H-L Hung, Y-F Huang, PAPR reduction in OFDM system using differential evolution-based partial transmit sequences scheme. IET Commun. 6(11), 1483-1488 (2012)

10. N Taspinar, A Kalinli, M Yildrim, Partial transmit sequences for PAPR reduction using parallel tabu search algorithm in OFDM systems. IEEE Communications Letter 15(9), 974-976 (2011)

11. J Tellado, Peak to Average Power Reduction for Multicarrier Modulation (Stanford University, Ph.D. dissertation, 2000)

12. Y Wang, W Chen, C Tellambura, Genetic algorithm based nearly optimal peak reduction tone set selection for adaptive amplitude clipping PAPR reduction. IEEE Trans. Broadcast. 58(3), 462-471 (2012)

13. J-C Chen, M-H Chiu, Y-S Yang, C-P Li, A suboptimal tone reservation algorithm based on cross-entropy method for PAPR reduction in OFDM systems. IEEE Trans. Broadcast. 57(3), 752-757 (2011)

14. H Li, T Jiang, Y Zhou, An improved tone reservation scheme with fast convergence for PAPR reduction in OFDM systems. IEEE Trans. Broadcast 57(4), 902-906 (2011)

15. J-C Chen, C-P Li, Tone reservation using near-optimal peak reduction tone set selection algorithm for PAPR reduction in OFDM systems. IEEE Signal Processing Letters 17(11), 933-936 (2010)

16. D Guel, J Palicot, Y Louet, Tone reservation technique based on geometric method for orthogonal frequency division multiplexing peak-to-average power ratio reduction. IET Commun. 4(17), 2065-2073 (2010)

17. S Gazor, R AliHemmati, Tone reservation for OFDM Systems by maximizing signal-to-distortion ratio. IEEE Trans. Commun. 11(2), 762-770 (2012)

18. J Tellado, JM Cioffi, PAR Reduction in Multicarrier Transmission System (ANSI Document, T1E1.4 Technical subcommittee, contribution number 97-367 (ANSI, Washington DC, 1997)

19. AR Mehrabian, C Lucas, A novel numerical optimization algorithm inspired from weed colonization. Ecological Informatics 1, 355-366 (2006)

20. GG Roy, S Das, P Chakraborty, PN Suganthan, Design of nonuniform circular antenna arrays using a modified invasive weed optimization algorithm. IEEE Trans. Antennas Propag. 59(1), 110-118 (2011)

21. S Karimkashi, AA Kishk, Invasive Weed Optimization and its Features in Electromagnetics. IEEE Trans. Antennas Propag. 58(4), 1269-1278 (2010)

22. Y-Y Bai, S Xiao, C Liu, B-Z Wang, A hybrid IWO/PSO algorithm for pattern synthesis of conformal phased arrays. IEEE Trans. Antennas Propag. 6(4), 2328-2332 (2013)

23. S Karimkashi, AA Kishk, G Zhang, Modelling of aperiodic array antennas using infinitesimal dipoles. IET Microwaves Antennas \& Propagation 6(7), 761-767 (2012)

24. A Foudazi, AR Mallahzadeh, Pattern synthesis for multi-feed reflector antennas using invasive weed optimisation. IET Microwaves, Antennas \& Propagation 6(14), 1583-1589 (2012)

25. J Kennedy, RC Eberhart, Swarm Intelligence (Morgan Kaufmann, San Mateo, 2001)

26. H-L Hung, Interference cancellation for HNNPSO multiuser detection of UWB systems over multipath fading channel. Springer Telecommunication Systems 12, 1-13 (2011)

27. DB Fogle, Evolutionary Computation: Toward a New Philosophy of Machine Intelligence, 2nd edn. (Piscataway, IEEE Press, 2000)

28. AJ Ratnaweera, SK Halgamuge, HC Watsom, Self-organizing hierarchical particle swarm optimizer with time-varying acceleration coefficients. IEEE Trans. Evolutionary Computation 8(3), 240-255 (2004)

29. Tellado, JM Cioffi, Peak Power Reduction for Multicarrier Transmission: Information Systems Laboratory (Stanford University, Stanford, 1999)

30. J Tellado, Multicarrier Modulation with Low PAPR (Kluwer, Massachusetts, 2000)

31. DW Lim, HS Noh, JS No, DJ Shin, Near optimal PRT set selection algorithm for tone reservation in OFDM systems. IEEE Trans. Broadcast. 54(3), 454-460 (2008)

doi:10.1186/1687-1499-2013-244

Cite this article as: Hung et al.: PAPR reduction of OFDM using invasive weed optimization-based optimal peak reduction tone set selection. EURASIP Journal on Wireless Communications and Networking 2013 2013:244 